\title{
Puzzling the 120-Cell
}

\section{Saul Schleimer and Henry Segerman}

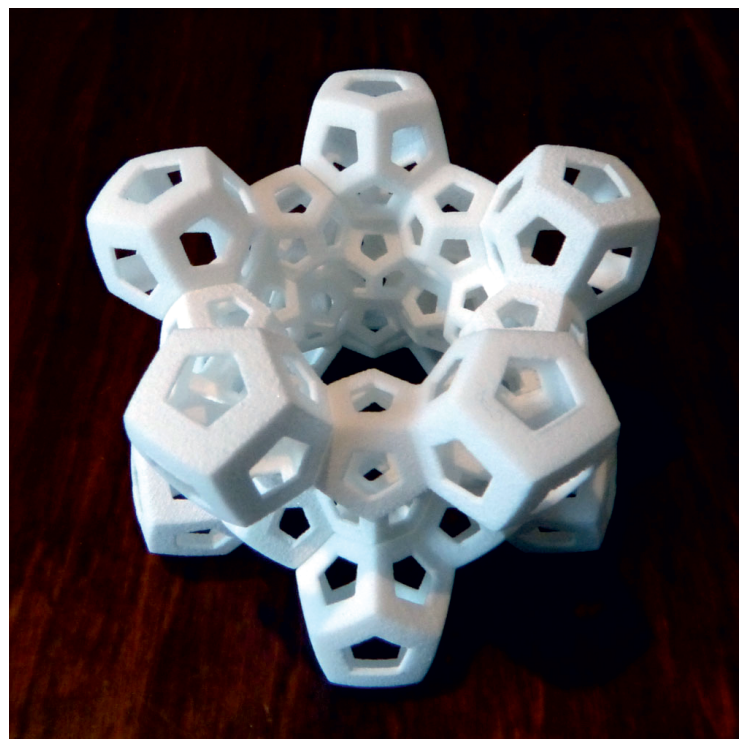

Figure 1. The Dc30 Ring, one of the simpler puzzles in Quintessence.

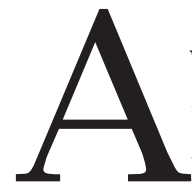

burr puzzle is a collection of notched wooden sticks [2, page xi] that fit together to form a highly symmetric design, often based on one of the Platonic solids. The assembled puzzle may have zero, one, or more internal voids; it may also have multiple solutions. Ideally, no force is required. Of course, a puzzle may violate these rules in various ways and still be called a burr.

The best known, and certainly largest, family of burr puzzles comprises what are collectively

Saul Schleimer is professor of mathematics at the University of Warwick. His email address is s.sch1eimer@warwick. ac.uk.

Henry Segerman is professor of mathematics at Oklahoma State University. His email address is segerman@math. okstate.edu.

All artwork is provided courtesy of the authors.

This article is in the public domain.

DOI: http://dx.doi.org/10.1090/noti1297 called the 6-piece burrs [5]. Another well-known burr, the star burr, is more closely related to our work. Unlike the 6-piece burrs, the six sticks of the star burr are all identical, as shown in Figure 2A. The solution is unique, and, once solved, the star burr has no internal voids. The solved puzzle is a copy of the first stellation of the rhombic dodecahedron; see Figure 2B.

The goal of this paper is to describe Quintessence: a new family of burr puzzles based on the 120-cell, a regular four-dimensional polytope. The puzzles are built from collections of six kinds of sticks, shown in Figure 3; we call these ribs, as they are gently curving chains of distorted dodecahedra.

In the following sections we review regular polytopes in low dimensions, sketch a construction of the dodecahedron, and discuss the three-sphere,

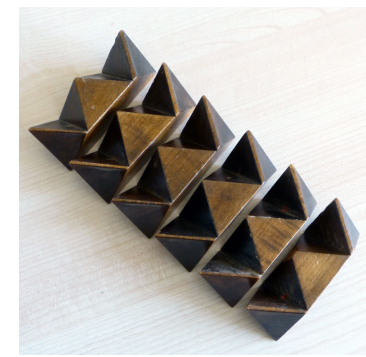

(A)

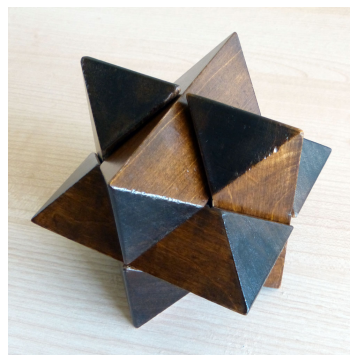

(B)
Figure 2. The star burr.

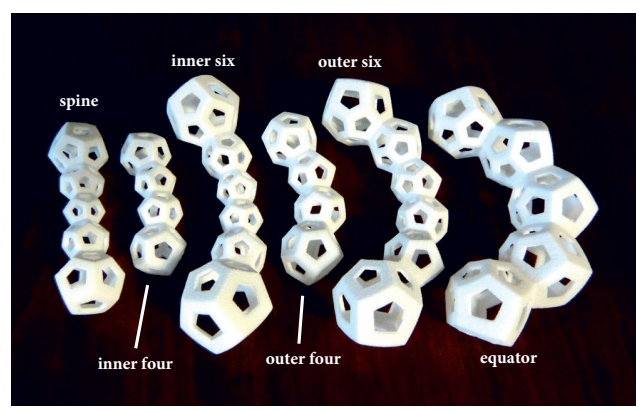

Figure 3. The six rib types. 

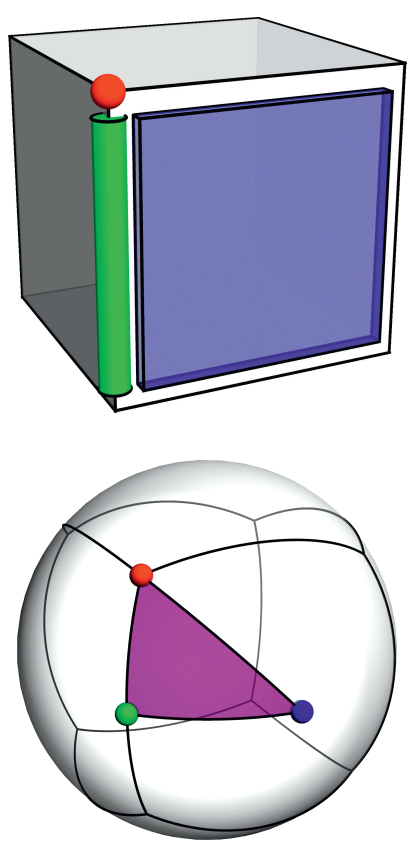

Figure 4. Flag for cube and corresponding spherical flag triangle.

quaternions, and stereographic projection. Viewing the binary dodecahedral group as a subgroup of the quaternions, we construct and then investigate the combinatorics of the 120-cell, focusing on how it decomposes into spheres and rings of dodecahedra. Finally, we lay out our choice of ribs, as influenced by the cell-centered stereographic projection. We use this to give a basic combinatorial restriction on the possible burr puzzles in Quintessence. One of the completed puzzles, the Dc30 Ring, is shown in Figure 1. The connection between the star burr and Quintessence is left as a final exercise for the intrigued reader.

\section{Polytopes}

We refer to [16] for an in-depth discussion of polytopes.

\section{Regular Polytopes}

Suppose that $P$ is a $k$-dimensional polytope. An ascending chain of faces $Q_{0} \subset Q_{1} \subset \cdots \subset Q_{k-1} \subset$ $Q_{k}=P$ is called a flag of $P$ if $Q_{\ell}$ has dimension $\ell$. See Figure 4 (top) for a picture of one of the forty-eight flags of the cube.

Let $\operatorname{Sym}(P)$ be the group of rigid motions (and reflections) preserving $P$ setwise. We call elements of $\operatorname{Sym}(P)$ the symmetries of $P$.

Definition 1. A polytope $P$ is regular if for any pair of its flags, $F$ and $G$, there is a symmetry $\phi \in$ $\operatorname{Sym}(P)$ with $\phi(F)=G$.

It follows that all facets of a regular polytope are congruent and are themselves regular.

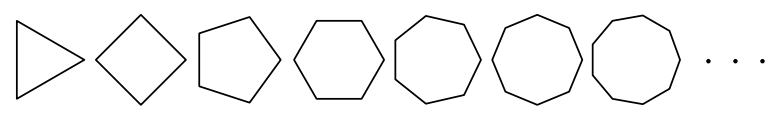

Figure 5. The regular polygons.

Suppose $P$ is regular. Define $p=\operatorname{center}(P)$ to be the average of the vertices of $P$. Since $\operatorname{Sym}(P)$ permutes the vertices of $P$, it fixes $p$. Since $\operatorname{Sym}(P)$ sends any flag to any other, the same is true of the vertices. So the vertices are all the same distance from $p$. Thus $p$ is a circumcenter: $P$ is circumscribed by the sphere $S_{P}$ centered at $p$ and running through the vertices of $P$. If we project $\partial P$ from $p$ outwards to $S_{P}$, we obtain a symmetrical spherical tiling $\mathcal{T}_{P}$.

Conversely, when we are constructing an $n$ dimensional regular polytope $P$, our first move is to build a spherical tiling $\mathcal{T}_{P}$ on $S^{n-1}$.

Definition 2. Suppose that $P$ is regular and $F=$ $\left\{Q_{i}\right\}$ is a flag in $P$. Then the flag polytope $Q_{F}$ is the convex hull of the centers of the $Q_{i}$. The spherical flag polytope is the radial projection of $Q_{F}-p$ to $S_{P}$. See Figure 4 (bottom).

If $P$ is regular, then all of its spherical flag polytopes are congruent.

\section{Constructions}

There are four infinite families of regular polytopes; each family is associated with a topological operation. The regular polygons live within the unit disk in the plane and are associated to covering maps of the circle by itself. See Figure 5.

Next, there are three families of regular polytopes that exist in all dimensions. Each member of a family is obtained from the previous member by a geometric operation. The simplices are cones, the cubes are products (with an interval), and the cross-polytopes are suspensions, that is, a double-cone.

Figure 6 shows the first several members of each family. In dimension two these are the triangle, square, and diamond, respectively. The fifth

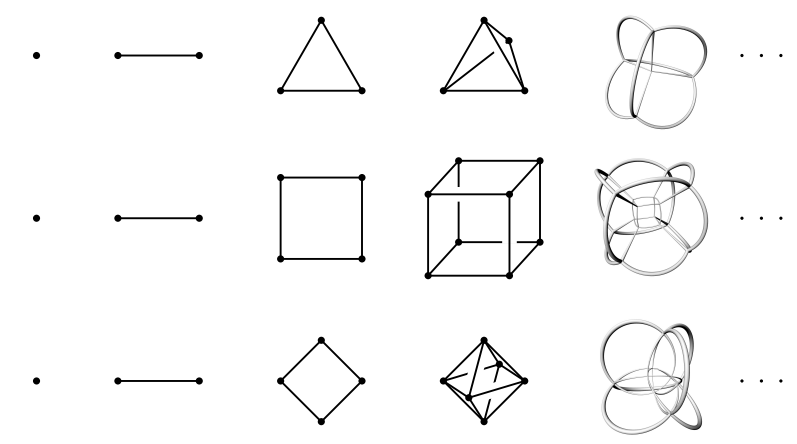

Figure 6. The first five simplices, cubes, and cross-polytopes. 

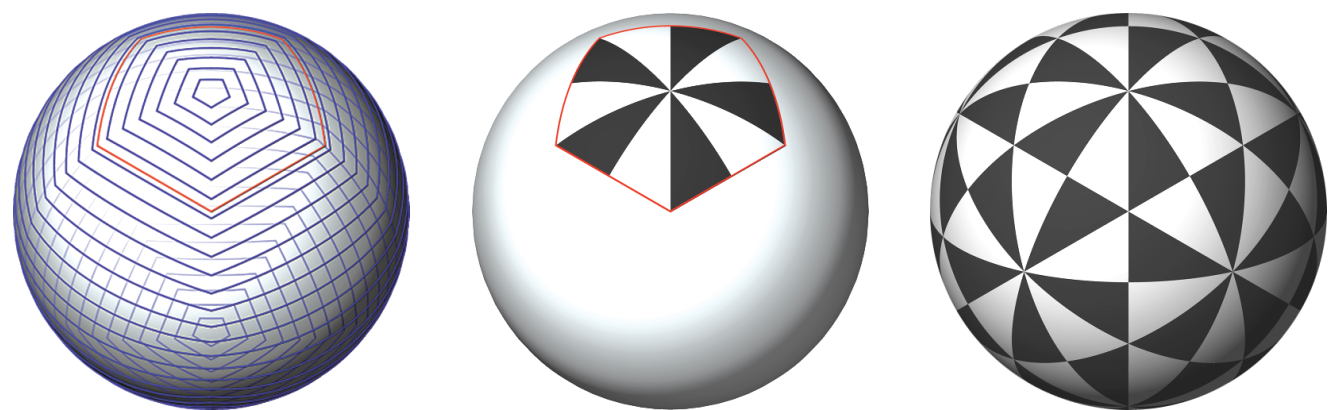

Figure 7. Left: The continuity argument. Middle: Dividing the pentagon into five right-handed spherical flags (in black) and five left-handed ones. Right: The tiling $\mathcal{T}$.

column shows the stereographic projections of the spherical tilings for the four-dimensional members of each family. These cannot be drawn in threedimensional spaces so we instead radially project their boundaries to $S^{3}$ and then stereographically project to $\mathbb{R}^{3}$. This technique is explained in [10]. For the convenience of the reader, we repeat the definition of stereographic projection below.

The four families give almost all of the regular polytopes; see [8, p. 143] for proofs of the following.

Lemma 3. The simplex, cube, and cross-polytope are regular. The cube and the cross-polytope are dual; the simplex is self-dual. In dimensions three and higher these three polytopes are distinct.

Theorem 4. There are exactly five regular polytopes not in one of the four families. These are, in dimension three, the dodecahedron and icosahedron (dual) and, in dimension four, the 24-cell (self-dual), and the 120-cell and 600-cell (dual).

\section{Dodecahedron}

The dodecahedron exists for more subtle reasons than those for the existence of the polygons, simplices, cubes, or cross-polytopes. As such it has many constructions; the earliest seems to be Proposition 17 in Book 13 of Euclid's Elements [7]. See [15] for one historical account of the five Platonic solids.

We sketch an indirect construction of the dodecahedron $D$ that has two advantages. The argument finds the symmetry group $\operatorname{Sym}(D)$ along the way. It also generalizes to all other regular tessellations of the sphere, the Euclidean plane, and hyperbolic plane.

By continuity, for any angle $\theta \in(3 \pi / 5,7 \pi / 5)$ there is a regular spherical pentagon $P \subset S^{2}$ with all angles equal to $\theta$. See Figure 7 (middle). Thus we may take $\theta$ equal to $2 \pi / 3$.

Adding a vertex at the center and at the midpoints of the edges, we divide $P$ into ten spherical flag triangles. These alternate between being right- and left-handed; all have internal angles $(\pi / 2, \pi / 3, \pi / 5)$. See Figure 7 (middle). These three angles appear at the edge, vertex, and center of $P$. Let $T_{R}\left(T_{L}\right)$ be one of the right-handed (left-handed) spherical flag triangles. Note that there are rotations of $S^{2}$ matching the edges of $T_{R}$ and $T_{L}$ in pairs.

The celebrated Poincaré polygon theorem [6, Theorem 4.14] now implies that copies of $T_{R}$ and $T_{L}$ give a tiling $\mathcal{T}$ of $S^{2}$, shown in Figure 7 (right). Poincaré's theorem also implies that $\operatorname{Sym}(\mathcal{T})$ is transitive on the triangles of $\mathcal{T}$ and that any local symmetry extends to give an element of $\operatorname{Sym}(\mathcal{T})$.

By Girard's formula [4, Equation 2.11], the area of a spherical triangle with interior angles $A, B, C$ is $A+B+C-\pi$. Thus the area of $T_{R}$ is

$$
\pi \cdot(1 / 2+1 / 3+1 / 5)-\pi=\pi / 30 \text {. }
$$

Since the area of $S^{2}$ is $4 \pi$, we deduce that the tiling $\mathcal{T}$ contains 120 triangles.

Definition 5. We partition $\mathcal{T}$ into copies of $P$ to obtain the tiling $\mathcal{T}_{D}$; this has 12 pentagonal faces, $12 \cdot 5 / 2=30$ edges, and $12 \cdot 5 / 3=20$ vertices. We take the convex hull (in $\mathbb{R}^{3}$ ) of the vertices of $\mathcal{T}_{D}$ (in $S^{2}$ ) to obtain $D$, the dodecahedron.

We end this section by examining the symmetries of $\mathcal{T}$.

Lemma 6. The group $\operatorname{Sym}(\mathcal{T})$ has order 120; the orientation-preserving subgroup $\mathcal{D}=\operatorname{Sym}^{+}(\mathcal{T})$ has order 60 . The tiling $\mathcal{T}$ is invariant under the antipodal map.

Corollary 7. The group $\mathcal{D}$ contains

- the identity,

- 12 face rotations through angle $2 \pi / 5$,

- 20 vertex rotations through angle $2 \pi / 3$,

- 12 face rotations through angle $4 \pi / 5$, and

- 15 edge rotations through angle $\pi$.

Proof. For any vertex $p$ of $\mathcal{T}$ of degree $2 d$ we obtain a cyclic subgroup $\mathbb{Z} / d \mathbb{Z}$ in $\mathcal{D}$. By the second part of Lemma 6 the vertex $p$ and its antipode $q$ give rise to the same subgroup. Thus we may count elements of $\mathcal{D}$ by always restricting to those rotations through an angle of $\pi$ or less. Counting the symmetries obtained this way gives 60 ; by the first part of Lemma 6 there are no others. 


\section{Four-Space and Quaternions}

In this section we recall the quaternions, the three-sphere, and stereographic projection. See also [4, Chapter 6], [14, Section 2.7], or [3, Part II]. The quaternions bridge the gap between the algebra of certain groups and the geometry of four-dimensional space. The three-sphere is the natural home of the spherical 120-cell.

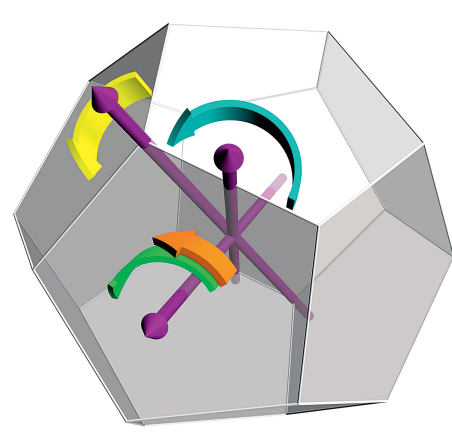

Due to the physiology of the human eye, we only ever see twodimensional images. The brain instinctively interprets some of these as representing threedimensional objects but is not equipped to deal with higher

Figure 8. Rotational dimensions. Hence symmetries of the we do not attempt dodecahedron. to draw any native pictures of four-dimensional objects. Instead, we use stereographic projection to transport objects from the three-sphere into three-dimensional space, where they can be seen with human eyes [10].

\section{The Quaternions}

The real numbers $\mathbb{R}$, being one-dimensional, can be augmented by adding $i=\sqrt{-1}$ to obtain the two-dimensional complex numbers $\mathbb{C}$. In very similar fashion Hamilton augmented $\mathbb{C}$ to obtain the quaternions $\mathbb{R}$. Let $\langle 1, i, j, k\rangle$ be the usual orthonormal basis for $\mathbb{R}^{4}$. We take $\mathbb{\|}=\mathbb{R} \oplus \mathbb{I}$, where $\mathbb{I}=i \mathbb{R} \oplus j \mathbb{R} \oplus k \mathbb{R}$ is the subspace of purely imaginary quaternions. Following Hamilton we endow $\mathbb{U}$ with the relations

$$
i^{2}=j^{2}=k^{2}=i j k=-1 \text {. }
$$

Since $\mathbb{U}$ is identical to $\mathbb{R}^{4}$ as a real vector space, there is a copy of the three-sphere inside the quaternions, namely, $S^{3}=\{q \in \mathbb{U}:|q|=1\}$ equipped with the induced metric. The function from $S^{3}$ to itself taking $p$ to $-p$ is called the antipodal map. When $L \subset \mathbb{Q}$ is a linear subspace of dimension one, two, or three, the intersection $L \cap S^{3}$ is a pair of antipodal points, a great circle, or a great sphere, respectively. We call the antipodal points 1 and -1 , as they lie in $S^{3}$, the south and north poles, respectively. We call $S_{\mathbb{I}}^{2}=S^{3} \cap \mathbb{I}$ the equatorial great sphere. See Figure 10 for a depiction of how several great circles among $1, i, j, k$ lie inside $S^{3}$.

\section{The Unit Quaternions}

The points of the three-sphere, the unit quaternions, form a group under quaternionic multiplication. Again, we see how the group structure and geometry of $S^{3}$ are tightly intertwined, as follows.

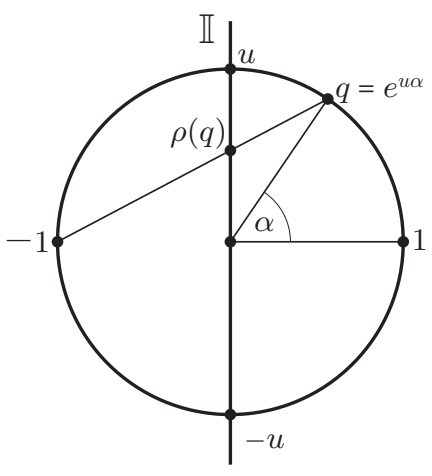

Figure 9. Stereographic projection from $S^{1}-\{-1\}$ to $\mathbb{I}$.

Lemma 8. The left and right actions of $S^{3}$ on $\mathbb{\square}$ are via orientation-preserving isometries. The same holds for the three-sphere's action on itself.

We can now parameterize great circles in $S^{3}$ through the identity. For any $u \in S_{\text {II }}^{2}$ define $L_{u}=\langle 1, u\rangle$ to be the corresponding plane in $\mathbb{H}$. The intersection $L_{u} \cup S^{3}$ is thus a great circle $C_{u}$. We parameterize $C_{u}$ by sending $\alpha \in \mathbb{R}$ to the point

$$
e^{u \alpha}=\cos \alpha+u \cdot \sin \alpha .
$$

Lemma 9. For any pure imaginary $u \in S_{\mathrm{I}}^{2}$ and for any $\alpha, \beta \in \mathbb{R}$ we have $e^{u \alpha} e^{u \beta}=e^{u(\alpha+\beta)}$. Thus $\left\{e^{u \alpha}\right\}$ is a one-parameter subgroup of $S^{3}$. Also, the spherical distance between 1 and $e^{u \alpha}$ is $\alpha$, for $\alpha \in$ $[0, \pi]$.

This gives a parameterization of $S^{3}$, as follows.

Lemma 10. For any $q \in S^{3}-\{ \pm 1\}$ there is a unique $u \in S_{\mathrm{I}}^{2}$ and a unique $\alpha \in(0, \pi)$ so that $q=e^{u \alpha}$.

\section{Stereographic Projection}

We define stereographic projection $\rho: S^{3}-\{-1\} \rightarrow \mathbb{I}$ by

$$
\rho(q)=\frac{\sin (\alpha)}{1+\cos (\alpha)} \cdot u
$$

with $q=e^{u \alpha}$ as in Lemma 10. See Figure 9 for a cross-sectional view. Note that $\rho$ sends the south pole to the origin, fixes the equatorial sphere $S_{\mathrm{I}}^{2}$ pointwise, and sends the north pole to "infinity." The one-parameter subgroup $e^{u \theta}$ is sent to the straight line in the direction of $u$. Figure 10 shows the result of applying stereographic projection to various great circles connecting $1, i, j, k$ inside $S^{3}$.

\section{Mapping to $\mathrm{SO}(3)$}

Recall that $\mathrm{SO}(3)$ is the group of three-by-three orthogonal matrices with determinant one. Taking $\langle i, j, k\rangle$ as a basis for $\mathbb{I}$, we identify $\mathrm{SO}(3)$ with Isom $_{0}^{+}(\mathrm{I})$, the group of orientation-preserving isometries of II fixing the origin. 


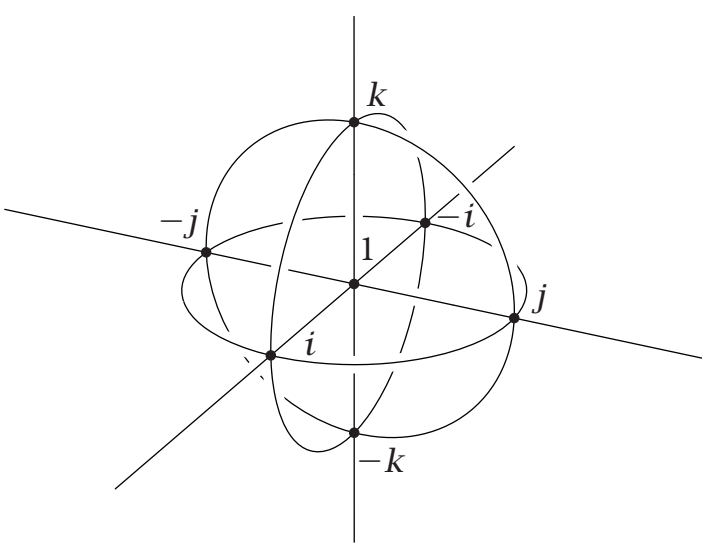

Figure 10. Several great circles connecting $1, i, j, k$, shown after stereographic projection to $\mathbb{R}^{3}$.

In Lemma 8 we discussed the left and right

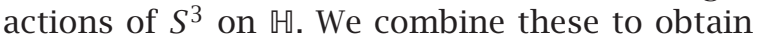
the twisted action: for $q \in S^{3}$ define $\phi_{q}: \mathbb{U} \rightarrow \mathbb{\sharp}$ by $\phi_{q}(p)=q p q^{-1}$. The twisted action is again via isometries. Note that the action preserves $\mathbb{R} \subset \mathbb{} \mathbb{\mathbb { N }}$ pointwise. Thus it preserves $\mathbb{I} \subset \mathbb{U}$ setwise. We define $\psi_{q}: \mathbb{I} \rightarrow \mathbb{I}$ by $\psi_{q}=\phi_{q} \mid \mathbb{I}$ and deduce the following.

Lemma 11. The map $\psi_{q}$ is an element of $\mathrm{SO}(3)$. The induced map $\psi: S^{3} \rightarrow \mathrm{SO}(3)$ is a group homomorphism.

We need an explicit form of $\psi$, discovered independently by Gauss, Rodrigues, Cayley, and Hamilton [12, p. 21].

Lemma 12. For $q= \pm e^{u \alpha}$ the isometry $\psi_{q}$ is a rotation of $\mathbb{I}$ about the direction $u$ through angle $2 \alpha$. Thus $\psi: S^{3} \rightarrow \mathrm{SO}(3)$ is a double cover.

Definition 13. If $G \subset \mathrm{SO}(3)$ is a group, then we call $G^{*}=\psi^{-1}(G)$ the binary group corresponding to $G$.

\section{The 120-Cell}

It is time to construct the 120-cell. We could use a continuity argument to build a spherical dodecahedron in $S^{3}$ with all dihedral angles equal to $2 \pi / 3$. The Poincaré polyhedron theorem would then produce a tiling of $S^{3}$; regularity of the tile implies regularity of the tiling. Taking the convex hull of the vertices would give the 120-cell. However, computing the number of cells would require computing the volume of the spherical flag polytope, a highly nontrivial task. Also, it is crucial for us to see how the binary dodecahedral group $\mathcal{D}^{*}$ lies inside the symmetry group of the 120-cell. Thus we give a more explicit construction. We refer to $[1,12,13]$ as very useful commentaries on the 120-cell.

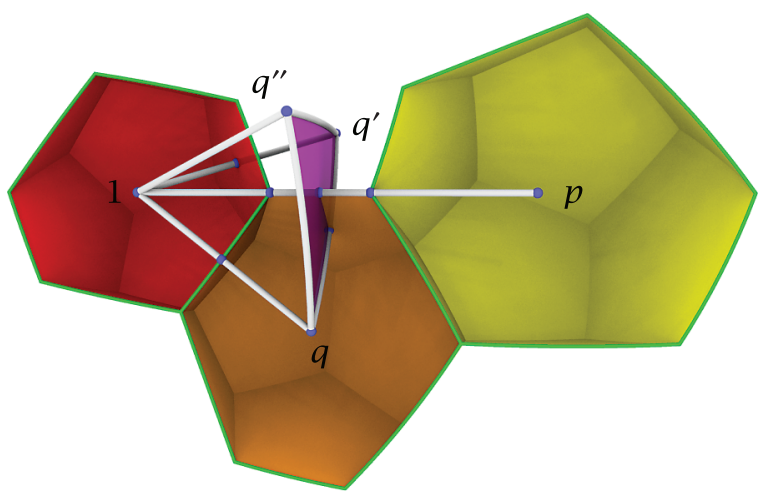

Figure 11 . Three dodecahedral cells of the tiling $\mathcal{T}_{120}$, each chopped in half. The purple triangle meeting the orange cell has vertices at $q, q^{\prime}$, and $q^{\prime \prime}$; it bisects the geodesic connecting 1 and $p$.

\section{Voronoi Cells}

Suppose $V$ is a finite set of points in a metric space $X$. The Voronoi cell about a point $q \in V$ is the set $\operatorname{Vor}(q)=\left\{r \in S^{3} \mid\right.$ for all $\left.p \in V, d_{X}(q, r) \leq d_{X}(p, r)\right\}$. Let $\mathcal{D} \subset \mathrm{SO}(3)$ be the group of orientationpreserving symmetries of the dodecahedron $D$, as listed in Corollary 7 . Let $\mathcal{D}^{*} \subset S^{3}$ be the corresponding binary dodecahedral group of 120 elements. Let $\mathcal{T}_{120}$ be the tiling of the three-sphere by the cells $\left\{\operatorname{Vor}(q) \mid q \in \mathcal{D}^{*}\right\}$.

Lemma 14. The left action of $\mathcal{D}^{*}$ on $\mathcal{T}_{120}$ is transitive on the three-cells. The twisted action of $\mathcal{D}^{*}$ fixes Vor(1) setwise.

Lemma 15. Each cell $\operatorname{Vor}(q)$ is a regular spherical dodecahedron with dihedral angle $2 \pi / 3$.

Proof. Figure 11 shows five points of $\mathcal{D}^{*}$ : namely, the identity 1 , a vertex rotation $p$, and the three face rotations $q, q^{\prime}$, and $q^{\prime \prime}$ about the faces immediately incident on the vertex. We also see three of the corresponding Voronoi cells, cut in half. Some delicate spherical trigonometry proves that $\operatorname{Vor}(1)$ is disjoint from $\operatorname{Vor}(p)$. Similar computations prove that $\operatorname{Vor}(1)$ meets only $\operatorname{Vor}(q)$ and its translates under the twisted action. Finally, the purple triangle in Figure 11 is equiangular, so $\operatorname{Vor}(q)$ (and thus Vor(1)) has the correct dihedral angle.

Remark 16. Applying the above construction to the cube does not give rise to a regular spherical cube; the vertex rotations are too close to the identity in $S^{3}$. The Voronoi cells are instead truncated cubes.

For the regular tetrahedron the vertex and face rotations are the same distance from the identity. Thus the Voronoi cells are regular spherical octahedra which tile the 24-cell.

Definition 17. The 120-Cell is the convex hull, taken in $\mathbb{U}$, of the vertices of $\mathcal{T}_{120}$. 


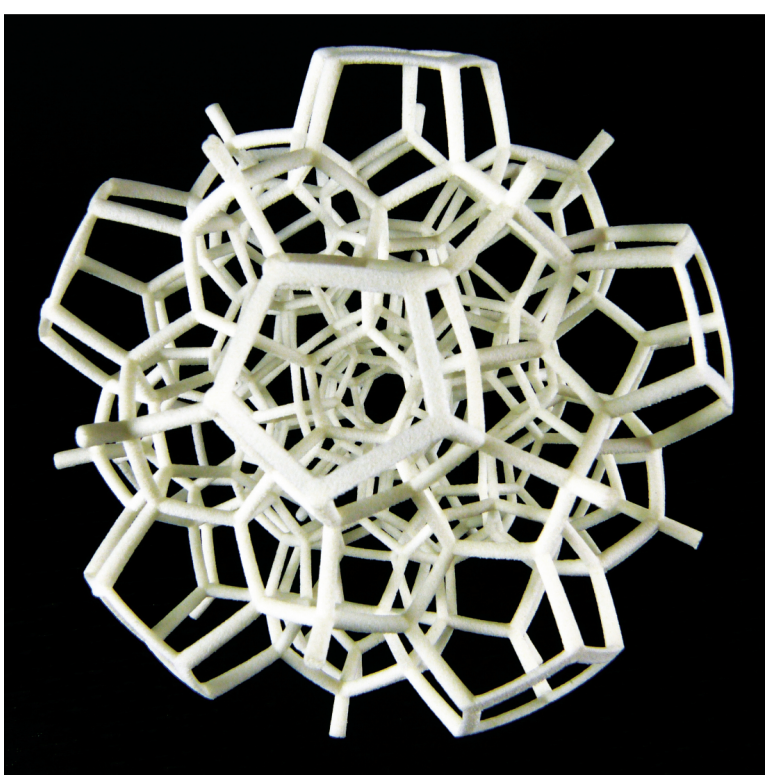

Figure 12. The southern half of the one-skeleton of $\mathcal{T}_{120}$, after cell-centered stereographic projection to $\mathbb{R}^{3}$. See also [13, color plate].

Theorem 18. The 120-cell is a regular polytope.

Proof. We must show that the group $\operatorname{Sym}\left(\mathcal{T}_{120}\right)$ acts transitively on the 14, 400 spherical flag tetrahedra of $\mathcal{T}_{120}$. Using the left action of $\mathcal{D}^{*}$ we may move any such into Vor(1). Using the twisted action we can move any right-handed spherical flag tetrahedron inside Vor(1) to any other. Now, since $\mathcal{T}$ is invariant under the antipodal map (Lemma 6) we deduce that $\mathcal{D}^{*}$ is fixed setwise by quaternionic conjugation. Since this conjugation is the product of three reflections in $\mathbb{t}$, it is orientation reversing in $S^{3}$.

See Figure 12 for a picture of the stereographic projection of the southern half of the one-skeleton of the spherical 120-cell.

\section{Combinatorics of the 120-cell}

With the 120-cell in hand, we turn to the combinatorics of the spherical tiling $\mathcal{T}_{120}$.

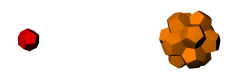

(A) 0

(B) $\pi / 5$

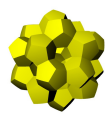

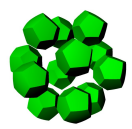

(D) $2 \pi / 5$

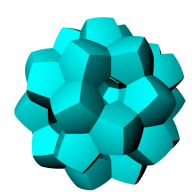

(E) $\pi / 2$
Figure 13. The five layers in the southern hemisphere, ordered by their spherical distance from the south pole. The colors of the cells follow the convention of Figure 8.

\section{Layers of Dodecahedra}

The cells of $\mathcal{T}_{120}$ divide into spherical layers, ordered by their distance from the identity element in $S^{3}$. Following our conventions, the identity lies at the south pole of $S^{3}$. Figure 13 displays the stereographic projections of the first five layers, expanding from the south pole out to the equatorial great sphere. The next four layers, nesting down to the north pole, are not shown. See Proposition 19 for more details.

\section{Rings of Dodecahedra}

Suppose that $q \in \mathcal{D}^{*}$ is the lift of a face rotation $A \in \mathcal{D}$ of angle $2 \pi / 5$. Let $R=\langle q\rangle<\mathcal{D}^{*}$ be the resulting cyclic group of order ten. Note that $R$ has twelve right cosets in $\mathcal{D}^{*}$. We call the cosets rings because each corresponding union of spherical dodecahedra forms a solid torus in $S^{3}$ (see Figure 14). We give the rings the following names: $R$ is the spinal ring, $R^{\mathrm{eq}}$ is the equatorial ring (having all elements at distance $\pi / 2$ from the south pole), $R_{0}^{\text {in }}$ to $R_{4}^{\text {in }}$ are the inner rings (each incident to the spine), and $R_{0}^{\text {out }}$ to $R_{4}^{\text {out }}$ are the outer rings (each incident to the equator).

Proposition 19. The rings meet the spherical layers of $\mathcal{T}_{120}$ as follows.

\begin{tabular}{ccccccc}
\multicolumn{2}{c}{ distance } & rotation type & \# cells & spinal & equatorial & inner \\
\hline 0 & identity & 1 & 1 & 0 & 0 & 0 \\
$\pi / 5$ & face & 12 & 2 & 0 & 2 & 0 \\
$\pi / 3$ & vertex & 20 & 0 & 0 & 2 & 2 \\
$2 \pi / 5$ & face & 12 & 2 & 0 & 0 & 2 \\
$\pi / 2$ & edge & 30 & 0 & 10 & 2 & 2 \\
$3 \pi / 5$ & face & 12 & 2 & 0 & 0 & 2 \\
$2 \pi / 3$ & vertex & 20 & 0 & 0 & 2 & 2 \\
$4 \pi / 5$ & face & 12 & 2 & 0 & 2 & 0 \\
$\pi$ & identity & 1 & 1 & 0 & 0 & 0
\end{tabular}

The column titled "\# cells" counts the number of cells in the named spherical layer.

See also [11].

Remark 20. The Hopf fibration is the partition of $S^{3}$ into cosets of the one-parameter subgroup $\{\exp (i \alpha)\}$. After a rotation, we see that the cosets of $R$ give a combinatorial Hopf fibration: they divide the 120-cell into twelve rings of ten dodecahedra each. The centers of the rings lie on twelve great circles of the Hopf fibration. Note also that the quotient space of the Hopf fibration is homeomorphic to $S^{2}$. In similar fashion there is a kind of combinatorial map from the 120-cell to the dodecahedron, sending rings to faces.

\section{Rings to Ribs}

In this section we describe the ribs of Quintessence: a collection of physical pieces that combine in various ways to produce burr puzzles. The ribs are shown in Figure 2; they are constructed via stereographic projection as applied to (parts of) the rings of spherical dodecahedra. 


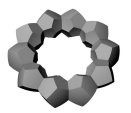

(A)

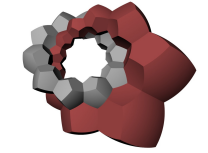

(B)

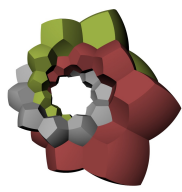

(C)

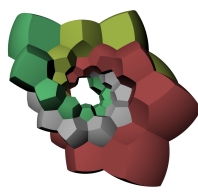

(D)

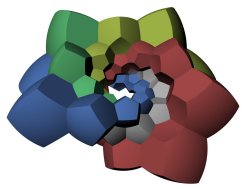

(E)

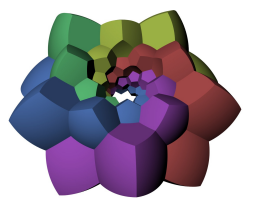

(F)

Figure 14. Rings of dodecahedra. (A) shows the equatorial ring. Figures (B) through (F) show the outer rings wrapping around it.

Following our notation above we have:

$$
\frac{d \rho}{d \alpha}=\frac{1}{1+\cos (\alpha)} \cdot u \text {. }
$$

Note that if $e^{u \alpha}$ is near the south pole, then $\alpha$ is close to zero and stereographic projection shrinks objects by a factor of approximately two. If $e^{u \alpha}$ is near the equatorial sphere, then $\alpha$ is close to $\pi / 2$. In this case stereographic projection leaves sizes essentially unchanged. However, if $e^{u \alpha}$ approaches the north pole, then $\alpha$ approaches $\pi$ and sizes blow up.

All of the calculations so far have been dimensionless. Now we wish to physically construct, say by $3 \mathrm{D}$ printing, our puzzle pieces. So we must choose a scale $\lambda$, say in millimeters, corresponding to a unit distance in $\mathbb{R}^{3}$. Several issues influence the choice of $\lambda$, but two are paramount. Large features are expensive; small features are fragile.

These two issues are in tension and lead to the general principle that features that are identical in $S^{3}$ should have sizes in reasonable ratio in $\mathbb{R}^{3}$ after projection. Here the features of the ribs are the dodecahedra. The principle tells us that we should not be using dodecahedra close to the north pole. On the other hand, sizes at the equator and at the south pole have an acceptable ratio of approximately two.

Accordingly, we remove from our rings any dodecahedra that lie strictly in the northern hemisphere, giving us the spine, the inner six ribs, and the outer six ribs. Experimentation shows that many interesting constructions require even shorter ribs; hence we also make the inner four ribs and the outer four ribs. These are the result of removing the two equatorial dodecahedra from the inner six and outer six. The equatorial ring can be printed as is, but again experimentation shows that more puzzles are possible if we break the equatorial ring into two ribs of five dodecahedra each. See Figure 3 as well as Figure 15.

With the spine and short ribs in hand, we can build, in $\mathbb{R}^{3}$, the stereographic projection of (almost) one-half of the 120-cell. We call the resulting puzzle the Dc45 Meteor; its construction is shown in Figure 16. The spine and ribs are arranged according to the combinatorial Hopf fibration (Remark 20). Since all dodecahedra near the south pole are retained and all dodecahedra

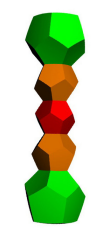

(A) Spine

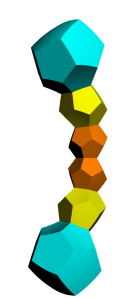

(B) Inner six

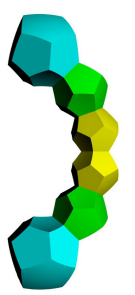

(C) Outer six

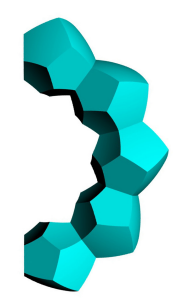

(D) Equator
Figure 15. The coloring of the cells is by layer and is consistent with Figure 13. We obtain the inner four and outer four ribs by deleting the equatorial cells.

near the north pole are discarded, the result looks very much like Figure 12.

It is not at all obvious that the puzzle can be constructed in Euclidean space using physical objects. However, when printed in plastic the Meteor is possible to assemble. When complete it holds together with no other support. Apparently a small amount of flex in the ribs is necessary; we have not been able to solve a similar puzzle when printed in a steel/bronze composite (the Dc30 Ring, shown in Figure 1).

It came as a surprise to us that there are numerous other burr puzzles using these ribs; most are not based on the combinatorial Hopf fibration [9]. However, there are significant combinatorial restrictions on the ribs that can be used in any burr puzzle. The following theorem is sharp, as shown by examples [9].

\section{Theorem 21.}

(1) At most six inner ribs are used in any puzzle.

(2) At most six outer ribs are used in any puzzle.

(3) At most ten inner and outer ribs are used in any puzzle.

Proof. The stereographic projection map $\rho$ is equivariant: $\rho$ transports the twisted action on $S^{3}$ to the $\mathrm{SO}(3)$ action on $\mathbb{R}^{3}$. That is, $\rho$ respects the $S^{2}$ symmetry about the south pole in $S^{3}$. Thus any two cells in a given layer (at fixed distance from the south pole) are congruent in $\mathbb{R}^{3}$. Also, any pair of cells in different layers are different due to the growth of $d \rho / d \alpha$. 


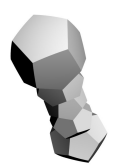

(A)

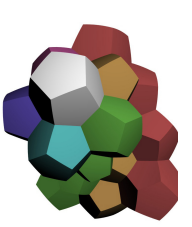

(G)

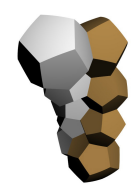

(B)

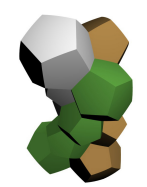

(C)

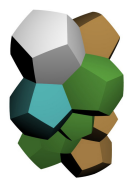

(D)

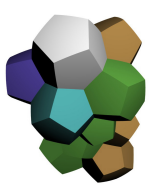

(E)

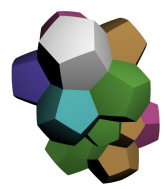

(F)

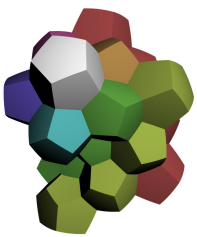

(H)

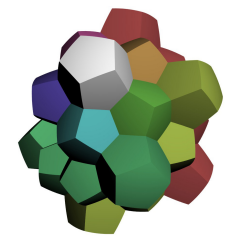

(I)

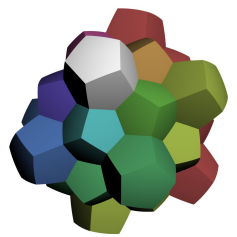

(J)

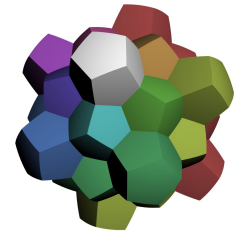

(K)

Figure 16. Building the Dc45 Meteor: Start with just the spine, in (A). One at a time add five copies of the inner four rib in (B) through (F). Then add five copies of the outer four rib, as in (G) through (K).

From the table in Proposition 19 we learn that each inner rib contains exactly two cells adjacent to the south pole. Next, column "\# cells" tells us there are exactly twelve such. Part (21) follows. We prove part (21) by examining the layer at distance $2 \pi / 5$, and we prove part (21) using the layer at distance $\pi / 3$. A color-coded guide is provided in Figures 13 and Figure 15.

\section{Acknowledgments}

We thank Robert Tang and Stuart Young for their insights into the combinatorics of the 120-cell.

\section{References}

[1] ARNAUd CHÉRITAT, Le 120, CNRS, 2012, images.math.cnrs.fr/Le-120.htm7

[2] Stewart Coffin, Geometric Puzzle Design, A K Peters Ltd., Wellesley, MA, 2007.

[3] John H. ConwAy and DereK A. SMith, On Quaternions and Octonions: Their Geometry, Arithmetic, and Symmetry, A K Peters Ltd., Natick, MA, 2003.

[4] Harold S. M. Coxeter, Regular Complex Polytopes, Cambridge University Press, London, 1974.

[5] Bill Cutler, A computer analysis of all 6-piece burrs, 1994, Www.CS.brandeis.edu/ storer/ JimPuzzles/BURR/000BURR/WEBPAGES/ CutlerComputerAnalysis,pdf

[6] David B. A. Epstein and Carlo Petronio, An exposition of Poincaré's polyhedron theorem, Enseign. Math. (2), 40(1-2):113-170, 1994.

[7] EuCLID, The Thirteen Books of Euclid's Elements Translated from the Text of Heiberg, Vol. I: Introduction and Books I, II. Vol. II: Books III-IX, Vol. III: Books X-XIII and Appendix, translated with introduction and commentary by Thomas L. Heath, 2nd ed., Dover Publications Inc., New York, 1956.

[8] L. Fejes Tóth, Regular Figures, a Pergamon Press Book, The Macmillan Co., New York, 1964.

[9] SAUl Schleimer and Henry Segerman, Quintessence manual, segerman.org/quintessence_manual.pd†
[10] Mathematics, Music, Art, Architecture, Culture, Tessellations Publishing, Phoenix, Arizona, 2012, pp. 103-110, archive.bridgesmathart.org/2012/ bridges2012-103 $\mathrm{h}+\mathrm{m}]$

[11] DunCAN M. Y. SOMMERVILLE, An Introduction to the Geometry of $n$ Dimensions, Dover Publications Inc., New York, 1958.

[12] JoHn STILlWELl, The story of the 120-cell, Notices Amer. Math. Soc., 48(1):17-24, 2001.

[13] John M. Sullivan, Generating and rendering fourdimensional polytopes, The Mathematica Journal, 1:76-85, 1991, torus.math.uiuc. edu/jms/Papers/ dodecaplex/

[14] William P. Thurston, Three-Dimensional Geometry and Topology, Vol. 1, volume 35 of Princeton Mathematical Series, Princeton University Press, Princeton, NJ, 1997.

[15] William C. WATERHOUSE, The discovery of the regular solids, Arch. History Exact Sci., 9(3):212-221, 1972.

[16] GÜNTER M. ZIEGLER, Lectures on Polytopes, volume 152 of Graduate Texts in Mathematics, Springer-Verlag, New York, 1995. 